(8) Die Beteiligung der Grünen an der CDU-geführten Koalition wurde von den Wählern nicht honoriert. Sie schafften nur knapp den Einzug in den neuen Landtag. In fast zwei Drittel der Gemeinden scheiterten sie an der Fünf-Prozent-Hürde. Von den 18- bis 29-Jährigen votierten nicht einmal halb so viele für die Grünen wie für die Piratenpartei.

(9) Die Piratenpartei ist der große Gewinner der Wahl. Da die Regierungszusammensetzung schon vor der Wahl praktisch feststand, konnten viele der mit den etablierten Parteien unzufriedenen jüngeren Saarländer ohne Risiko eine neue Alternative testen.

(10) Im Saarland besteht nunmehr ein vergleichsweise polarisiertes, symmetrisches Vielparteiensystem.

\title{
Die schleswig-holsteinische Landtagswahl vom 6. Mai 2012: SPD, Grüne und SSW bilden erste Dänen-Ampel
}

\section{Patrick Horst}

Die Schleswig-Holsteiner wurden im Mai 2012 erneut vorzeitig zu den Wahlurnen gerufen - anders als nach dem Bruch der Großen Koalition 2009 hatte das vorzeitige Ende der Wahlperiode diesmal jedoch im engeren Sinne keine politischen Gründe. Auslöser der Neuwahl war ein Urteil des Landesverfassungsgerichts, das Teile des Wahlgesetzes für verfassungswidrig erklärt hatte. Die Ein-Stimmen-Mehrheit der schwarz-gelben Koalition, die sich der für verfassungswidrig befundenen Regelung im Wahlrecht verdankte, ließ das Gericht unangetastet, trug dem Parlament jedoch eine Wahlrechtsreform und Neuwahl bis spätestens 30. September 2012 auf. ${ }^{1}$ CDU, FDP und SPD einigten sich im März 2011 auf einen Kompromiss, der die Zahl der Wahlkreise von 40 auf 35 reduziert und den vollständigen Ausgleich der Überhangmandate vorsieht, wodurch der mögliche Verzerrungseffekt des bisherigen Wahlrechts beseitigt wurde. ${ }^{2}$ Zeitgleich verständigten sich die politischen Kontrahenten auf den 6. Mai 2012 als Wahltermin. Der ersten schwarz-gelben Koalition seit Anfang der 1970er Jahre in Schleswig-Holstein war deshalb, wie sich zeigen sollte, nur ein kurzes Interregnum von zweieinhalb Jahren vergönnt.

1 Siehe Schleswig-Holsteinisches Landesverfassungsgericht, LVerfG 3/09, 30. August 2010. Zu einer eingehenden Diskussion der mit dem alten Wahlrecht verbundenen Probleme siehe Eric Linhart I Harald Schoen, Überhang- und Ausgleichsmandate in Schleswig-Holstein: Unklares Wahlrecht und Reformvorschläge, in: ZParl, 41. Jg. (2010), H. 2, S. 290 - 303.

2 Vgl. die Plenardebatte des Schleswig-Holsteinischen Landtages vom 24. März 2011 mit den dazugehörigen Drucksachen, PIPr. 17/45, S. 3789 - 3814. Die nur geringfügige Reduzierung der Wahlkreise beseitigte das Problem der Überhangmandate nicht grundsätzlich. Weil also nach wie vor eine Vergrößerung des Landtages durch Überhang- und Ausgleichsmandate möglich ist, wurde die Bestimmung über die Sollgröße von 69 Abgeordneten aus der Verfassung gestrichen und ins Wahlgesetz geschrieben. 


\section{Die Ausgangslage}

Die Ausgangslage für das amtierende schwarz-gelbe Bündnis war aus mehreren Gründen ungünstig - wenn nicht gar aussichtslos: Die CDU/CSU-FDP-Koalition im Bund, in deren „Windschatten“ die Koalition in Kiel im Oktober 2009 gebildet worden war" ${ }^{3}$, hatte einen miserablen Start erwischt, der vor allem die Freidemokraten in eine schwere Existenzkrise stürzte. Bei den Landtagswahlen in Nordrhein-Westfalen im Mai 2010 und in Baden-Württemberg zehn Monate später waren bereits zwei schwarz-gelbe Koalitionen abgewählt worden. In beiden Fällen hatten zusätzlich Versäumnisse des jeweiligen CDU-Landesverbandes zu den Wahlniederlagen beigetragen. ${ }^{4}$ Auch um die schleswig-holsteinische CDU war es nicht zum Besten bestellt. Der ursprünglich geplante Stabwechsel von Ministerpräsident Peter Harry Carstensen zu Fraktionschef Christian von Boetticher war im August 2011 an einer peinlichen „Lolita-Affäre“ gescheitert; dem als Spitzenkandidat in die Bresche gesprungenen Wirtschaftsminister Jost de Jager fehlte es im Lande an Bekanntheit und an einem signifikanten Profil. Die Wahlumfragen stellten der CDU regelmäßig knapp über 30, der FDP bis einen Monat vor der Wahl immer unter fünf Prozent in Aussicht. Hinzu kam, dass die Piratenpartei sich seit ihrem Einzug in das Berliner Abgeordnetenhaus im September 2011 auch in Schleswig-Holstein bei über fünf Prozent stabilisiert hatte. Nach ihrem Erfolg im Saarland im März 2012 erzielte sie sogar kurzzeitig zweistellige Werte in den schleswigholsteinischen Wahlumfragen, weshalb ihr Einzug in den Kieler Landtag kaum mehr in Frage stand. 5 Das machte eine Neuauflage der amtierenden Koalition noch unwahrscheinlicher, eröffnete aber immerhin der CDU ein Hintertürchen an die Macht: Sollte sie stärkste Fraktion im neuen Landtag werden und es für eine Koalition der SPD mit den Grünen - gegebenenfalls erweitert um den Südschleswigschen Wählerverband (SSW) - nicht reichen, so könnte ihr der Auftrag zur Regierungsbildung zufallen.

Auch eine Große Koalition, die 2009 noch an der unverträglichen „persönlichen Chemie“ zwischen Carstensen und dem SPD-Landesvorsitzenden Ralf Stegner gescheitert war, erschien wieder im Bereich des Möglichen. So wie Carstensen auf Seiten der CDU nicht erneut zur Wahl antrat, hatte auch die SPD im Zuge eines Mitgliederentscheids einen neuen Spitzenkandidaten nominiert: Anstelle ihres politisch wie persönlich stark polarisierenden Parteivorsitzenden hatte sie im Februar 2011 den Kieler Oberbürgermeister Torsten Albig auf den Schild gehoben. Er war zwar auch - ähnlich wie de Jager - nur etwa der Hälfte der Wähler in Schleswig-Holstein bekannt, hatte aber ein deutlich positiveres Persönlichkeitsprofil als Stegner und auch als sein Kontrahent um das Ministerpräsidentenamt: Während er hinsichtlich des ihm zugewiesenen Sachverstands gleichauf mit de Jager lag, hatte er bei den weicheren Eigenschaften der persönlichen Glaubwürdigkeit und Sympathie deutliche Pluspunkte im Wählerurteil zu verzeichnen. Die Entscheidung der SPD, nicht wieder mit

3 Dazu Patrick Horst, Die schleswig-holsteinische Landtagswahl vom 27. September 2009: Ministerpräsident auf Abruf kann nach vorgezogener Neuwahl schwarz-gelbe Wunschkoalition bilden, in: ZParl, 41. Jg. (2010), H. 2, S. $372-389$.

4 Siehe die Analysen von Ursula Feist / Hans-Jürgen Hoffmann, in: ZParl, 41. Jg. (2010), H. 4, S. 766 - 787, sowie von Oscar W. Gabriel / Bernhard Kornelius, in: ZParl, 42. Jg. (2011), H. 4, S. $784-804$.

5 Vgl. http://www.wahlrecht.de/umfragen/landtage/schleswig-holstein.htm (Abruf am 14. Juni 2012). 
Stegner anzutreten, war weise: Seit September 2011 lag Albig bei der hypothetischen „Direktwahlfrage" nach dem gewünschten Ministerpräsidenten immer weit vor de Jager. ${ }^{6}$

Auch die Grünen hatten sich nach der Landtagswahl 2009 neu aufgestellt und traten 2012 mit einem neuen Hoffnungsträger und Spitzenkandidaten an: dem 42-jährigen Schriftsteller Robert Habeck. Er gehörte einer neuen Generation an und hatte in der Vergangenheit für die koalitionspolitische Öffnung seiner Partei in Richtung der CDU plädiert. Für schwarz-grüne Bündnisse waren die Zeiten jedoch denkbar ungünstig: Nach dem Scheitern der ersten schwarz-grünen Koalition im Nachbarland Hamburg im November $2010^{7}$ hatten beide Parteien im Bund wie in den Ländern wieder die gegenseitige Abgrenzung verstärkt. Für die überwiegende Mehrheit der Grünen in Schleswig-Holstein war deshalb ausgemacht, dass sie den politischen Wechsel an der Seite der SPD herbeiführen wollten; auf dieses Koalitionssignal legten sie sich schließlich auch fest und banden damit ihren Spitzenkandidaten Habeck. Ungeachtet dieser Entscheidung für Rot-Grün strahlte Habeck persönlich ins Lager der Wähler von Union und FDP aus: Seine Wertschätzung bei allen Wählern war genauso hoch wie de Jagers und übertraf noch diejenige von Anke Spoorendonk (SSW) und Wolfgang Kubicki (FDP), die beide seit Jahrzehnten feste Größen der Landespolitik sind. ${ }^{8}$

Für die FDP war die Ausgangslage schwierig, aber nicht hoffnungslos. Zwar war auch sie im Sog des bundespolitischen Niedergangs im Verlauf des Jahres 2010 unter die Fünfprozenthürde gesunken; sie hatte jedoch mit Kubicki einen zugkräftigen Spitzenkandidaten, der es schon in der Vergangenheit immer wieder verstanden hatte, für ein unabhängiges, stärker sozialliberal ausgerichtetes Profil seiner Landespartei zu sorgen. Es bestanden eigentlich wenig Zweifel, dass es ihm auch diesmal wieder gelingen würde, mit markigen Äußerungen die Aufmerksamkeit der Medien auf sich zu ziehen und sich so - ähnlich wie Christian Lindner in Nordrhein-Westfalen - erfolgreich von der Bundespartei abzusetzen. Über ein vergleichbares Zugpferd verfügte die Linke dagegen nicht, so dass für sie die Ausgangslage nicht nur schwierig, sondern auch aussichtslos war. Da zudem diesmal mit den Piraten eine attraktive Alternative für Protestwähler bereitstand, war mit einem erneuten Einzug der Linken in den Landtag nicht zu rechnen.?

Die Leistungsbilanz der schwarz-gelben Koalition wurde von der Mehrheit der Bürger überwiegend kritisch bewertet. Im März und April 2012 zeigten sich etwa sechs von zehn Wählern unzufrieden mit der Arbeit der Landesregierung, wobei die Kritik an der FDP deutlich stärker ausfiel als an der CDU. Einen Hoffnungsschimmer für die Regierungsparteien bot allein die gute Konjunkturlage, die zu einer positiven Einschätzung der Wirtschaftsentwicklung führte: Etwa acht von zehn Schleswig-Holsteinern erwarteten eine gleichbleibende oder sogar eine verbesserte Wirtschaftslage in der Zukunft. CDU und FDP profitierten davon dennoch nicht: Zum einen lenkte die gute Konjunktur die Aufmerksamkeit auf andere Themen, allen voran die Situation im Schulbereich, wo das Hin und Her

6 Siehe Infratest dimap, LänderTREND Schleswig-Holstein, September 2011, Februar, März und April 2012; Forschungsgruppe Wahlen, Wahl in Schleswig-Holstein. Eine Analyse der Landtagswahl in Schleswig-Holstein vom 6. Mai 2012, Mannheim, Mai 2012, S. 21 - 25.

7 Dazu Patrick Horst, Die Wahl zur Hamburger Bürgerschaft vom 20. Februar 2011: Ehemalige „Hamburg-Partei“ erobert absolute Mehrheit zurück, in: ZParl, 42. Jg. (2011), H. 4, S. $724-744$.

8 Vgl. Forschungsgruppe Wahlen, a.a.O. (Fn. 6), S. 24.

9 Siehe „Links liegen gelassen“, in: Hamburger Abendblatt vom 12. April 2012, S. 14. 
zwischen dem acht- und neunjährigen Abitur für Unruhe gesorgt hatte. Zum anderen vermochten die Wähler zwischen der Konjunkturlage und der Leistung der Parteien zu trennen: Zwar lag die CDU in der Kompetenzbewertung auf den Gebieten der Wirtschafts- und der Finanzpolitik noch vor der SPD, aber nur denkbar knapp. Auf den anderen Politikfeldern hatten die Sozialdemokraten und die Grünen der CDU - teils deutlich - den Rang abgelaufen. In der Gesamtschau der Themenprioritäten, der Parteikompetenzen und der Persönlichkeitsfaktoren deutete alles auf einen politischen Wechsel hin, der über alle Umfragen hinweg auch von der Mehrheit der Wähler gewünscht wurde. ${ }^{10}$

\section{Der Wahlkampf}

Der Wahlkampf begleitete die Arbeit des 17. Schleswig-Holsteinischen Landtages fast von Beginn an: Mit dem Urteil des Landesverfassungsgerichts vom 30. August 2010 stand fest, dass spätestens bis zum Herbst 2012 Wahlen stattfinden mussten. Entsprechend früh setzten die Vorbereitungen der Parteien für den Wahlkampf ein: Anfang September erklärten auf Seiten der SPD zunächst der Kieler Oberbürgermeister Torsten Albig und wenige Tage später der Landesvorsitzende Ralf Stegner, dass sie ihre Partei als Spitzenkandidaten in den Wahlkampf führen wollten. Den daraufhin anberaumten Mitgliederentscheid entschied Albig Ende Februar 2011 mit 57 zu 32 Prozent für sich. ${ }^{11}$ Ebenfalls noch im September 2010 wählte die CDU ihren Fraktionschef Christian von Boetticher als Nachfolger von Peter Harry Carstensen zum Parteivorsitzenden, Anfang Mai 2011 dann auch zu ihrem Ministerpräsidentenkandidaten. Noch im August drängte ihn die Partei aber aufgrund einer tränenreich zugestandenen Liebesaffäre mit einer Minderjährigen zum Rücktritt. ${ }^{12}$ Wirtschaftsminister Jost de Jager war schnell als Ersatz zur Stelle. Die anderen Parteien nominierten ihre Spitzenkandidaten offiziell um die Jahreswende 2011 auf 2012: Kubicki trat für die FDP an, Habeck für die Grünen (auch wenn er nur auf Platz zwei der Landesliste hinter der Finanzexpertin Monika Heinold nominiert war), Spoorendonk für den SSW und Albig für die SPD. Die Linke ging mit ihrer Parteivorsitzenden Antje Jansen ins Rennen, die Piratenpartei mit Torge Schmidt.

Mit Beginn des Wahljahres 2012 positionierten sich die Parteien zunächst mit Blick auf ihre Koalitionssignale. ${ }^{13}$ Einmal mehr zeigte sich, wie sehr sie sich dabei nicht nur an ihren Wünschen, sondern zuvorderst an den Realitäten - also den laut Wahlumfragen erreichbaren Koalitionen - orientieren: Die CDU kündigte angesichts der akuten Umfrageschwäche der

10 Vgl. Forschungsgruppe Wahlen, a.a.O. (Fn. 6), S. 16 - 30; Infratest dimap, a.a.O. (Fn. 6).

11 Die restlichen zehn Prozent entfielen auf zwei weitere Kandidaten. Am Mitgliederentscheid beteiligten sich immerhin fast 70 Prozent der 19.200 schleswig-holsteinischen Parteimitglieder. Vgl. „Mitgliederentscheid: Schleswig-Holsteins SPD wählt Albig zum Spitzenkandidaten“, in: tagesspiegel.de vom 26. Februar 2011 (Abruf am 14. Juni 2012).

12 Siehe „Dossier: Christian von Boetticher: Feind, Todfeind, Parteifreund“, in: abendblatt.de vom 29. Dezember 2011 (Abruf am 21. Juni 2012).

13 Frank Decker hat den überzeugenden Vorschlag gemacht, von differenzierten Koalitionssignalen anstatt von Koalitionsaussagen zu sprechen. Vgl. ders., Koalitionsaussagen der Parteien vor Wahlen. Eine Forschungsskizze im Kontext des deutschen Regierungssystems, in: ZParl, 40. Jg. (2009), H. 2, S. 431 - 453; ders., Regieren im „Parteienbundesstaat“. Zur Architektur der deutschen Politik, Wiesbaden 2011, S. 105 - 130. 
FDP an, dass sie ohne Koalitionsaussage in die Wahl ziehen und nur für ihre eigene Stärke werben wolle. Dreier-Bündnissen erteilte de Jager aufgrund ihrer angeblichen Instabilität eine Absage - diese Aussage richtete sich mindestens so sehr gegen eine mögliche „DänenAmpel“ wie eine gegebenenfalls von der CDU geführte Dreierkonstellation. Für die Union kamen folglich nur die Grünen und die SPD als mögliche Koalitionspartner in Frage; diese erwiderten das positive Koalitionssignal der CDU jedoch nicht. Die Sozialdemokraten legten sich angesichts der günstigen Umfragewerte schon sehr früh auf Rot-Grün als erste Präferenz fest, die Grünen zögerten ihre Klarstellung noch bis in den März hinaus. ${ }^{14}$ Beide machten gegen Ende des Wahlkampfes auch deutlich, dass sie - sollte es für Rot-Grün nicht reichen - ein Bündnis mit dem SSW eingehen würden. Für kurzzeitige Erregung innerhalb der CDU sorgte deshalb die Ankündigung Spoorendonks im Februar, dass der SSW den Machtwechsel wolle und bereit sei, sich an einer rot-grünen Koalition zu beteiligen. ${ }^{15}$ Von den Christdemokraten, auch von Spitzenkandidat de Jager, wurde nun wieder das leidige, demokratietheoretisch unhaltbare Argument hervorgekehrt, der politische Minderheitenstatus der Dänen berechtige sie nicht, über die Regierungszusammensetzung im Lande zu entscheiden. ${ }^{16}$ Die FDP kämpfte vor allem um ihr Überleben und setzte alle Karten auf Spitzenkandidat Kubicki, der sich als Finanzminister ins Spiel brachte - unklar blieb jedoch welcher Regierungsformation. Die Liberalen verzichteten auf eine Koalitionsaussage, liebäugelten aber mit der Jamaika-Koalition. ${ }^{17}$ Für Erstaunen sorgten die Piraten, die sich auch eine Regierungsbeteiligung vorstellen konnten. ${ }^{18}$

Der Wahlkampf war eher arm an Inhalten und streitig geführten Auseinandersetzungen. Das hatte viel mit der Ausgangslage zu tun, die für die bisherigen Oppositionsparteien weit günstiger war als für die Regierungspartner. Für die SPD bestand folglich keine Notwendigkeit, scharfe Attacken gegen CDU und FDP zu führen. Dem Naturell von Spitzenkandidat Albig lag dies ohnehin fern. Für ihn war es die erfolgversprechendere Strategie, auf seinen Sympathiebonus zu setzen und inhaltlich im Ungefähren zu bleiben. Albig führte einen „Schmusewahlkampf"19 und warb mit dem Slogan „Mein Lieblingsland braucht gute Arbeit“ respektive „neue Energie“ und „starke Kinder“. Manch ein innerparteilicher Kritiker

14 Vgl. „SPD und CDU im Norden haben Grüne im Visier“, in: kn-online.de vom 29. Dezember 2011; „Spitzenkandidat Habeck: Für Grüne SPD erster Ansprechpartner“, in: ebenda vom 6. März 2012; „CDU-Spitzenkandidat de Jager: Dreier-Bündnisse instabil“, in: ebenda vom 23. März 2012; „Nord-Grüne streben Bündnis mit SPD an“, in: ebenda vom 26. März 2012 (Abruf am 10. April 2012).

15 Siehe „SSW will Machtwechsel“, in: ebenda vom 11. Februar 2012 (Abruf am 10. April 2012).

16 Vgl. „Schon wieder Streit um die Rolle des SSW“, in: kn-online.de vom 20. Februar 2012 (Abruf am 10. April 2012); „CDU attackiert SSW wegen geplanter ,Dänen-Ampel““, in: ebenda vom 25. April 2012 (Abruf am 3. Mai 2012). Die entscheidenden Argumente, warum der Minderheitenstatus der Dänen sie nicht zu Bürgern zweiter Klasse macht, hat Everhard Holtmann, Dürfen die das, wo sie doch Dänen sind? Über den Umgang mit Macht und Minderheiten in Deutschland, in: ZParl, 36. Jg. (2005), H. 3, S. 616 - 629, zusammengestellt.

17 Vgl. „Kubickis One-Man-Show“, in: Hamburger Abendblatt vom 12. März 2012, S. 12; „Kubicki hofft auf,Jamaika“", in: kn-online.de vom 27. Februar 2012 (Abruf am 10. April 2012).

18 Dazu „Piraten-Chef Nerz hält Regierungsbeteiligung für möglich“, in: kn-online.de vom 22. April 2012 (Abruf am 3. Mai 2012); Torge Schmidt im Interview: „Wir haben kein Problem damit, Kompromisse zu machen“, in: sueddeutsche.de vom 3. Mai 2012 (Abruf am 20. Juli 2012).

19 Joachim Krause, zitiert in: „Die Wahl der Spitzenkräfte“, in: Hamburger Abendblatt vom 10. April 2012, S. 14. 
sprach hinter vorgehaltener Hand von der „Schlafwagen-Strategie“ des Spitzenkandidaten. ${ }^{20}$ Dieser ließ sich von solcher Kritik jedoch nicht aus der Ruhe bringen und blieb konsequent bei seiner Linie, nur positive Nachrichten unter das Wahlvolk zu bringen: Den Kommunen wollte er wieder ausreichend Finanzmittel zur Verfügung stellen, für stärkere Investitionen in die Bildung und in Kindertagesplätze sorgen, schließlich auch die Energiewende voranbringen. Bei alldem sollte die Haushaltskonsolidierung fortgeführt und die landesverfassungsrechtlich vorgesehene „Schuldenbremse“, welche die Rückführung des strukturellen Haushaltsdefizits bis 2020 auf Null vorsieht, eingehalten werden. ${ }^{21}$

Der grüne Spitzenkandidat Habeck machte es Albig nach und punktete vor allem mit seiner „unverkrampften Art“22. Auch er vertrat offensiv die Strategie, „ausschließlich einen Positivwahlkampf“23 zu führen. Das zentrale Motto der grünen Wahlkampagne lautete „Für hier mit dir“. Auf den Plakaten war nur Habeck zu sehen: vor einem Windrad („Zieht wie Hechtsuppe“), im Watt („Jeder braucht watt zum Leben“), vor einer Schulklasse („Bankenkrise stoppen“) und vor der ausgelegten Ware eines Bio-Bauernhofes („Iss es, wie’s is“). ${ }^{24} \mathrm{Im}$ drei Wochen vor der Wahl präsentierten „100-Tage-Regierungsprogramm“ stellten die Grünen die Energie-, Klimaschutz- und Bildungspolitik in den Mittelpunkt. Sie forderten ein Energiewendeministerium, die Wiedereinführung von Subventionen für den Ökolandbau, die Rücknahme der geplanten Streichung von 300 Lehrerstellen und die Wiedereinführung der vollständigen Förderung von Schulen der dänischen Minderheit. Die Mehrkosten ab 2013 in Höhe von angeblich 20 Millionen Euro wollten die Grünen durch Umschichtungen im Haushalt sowie durch Bundesratsinitiativen zur Einführung einer Vermögensteuer und zur Rücknahme der Mehrwertsteuersenkung für Hotelübernachtungen kompensieren. Weitere Wahlversprechen beinhalteten die Verabschiedung eines Tariftreue- und eines Mindestlohngesetzes, die Ablehnung der Fehmarnbelt-Querung und die Absenkung des Wahlalters auf 16 Jahre. $^{25}$

Angesichts des wenig konfrontativen Wahlkampfstils der beiden größten Oppositionsparteien im Kieler Landtag sahen sich die Regierungsparteien in die unvorteilhafte Lage versetzt, selbst zum Angriff übergehen zu müssen. Zwar ist dies für einen Wahlkampf aus der Regierung heraus selten erfolgversprechend, dennoch hatte vor allem die CDU keine andere Wahl. Ab Anfang Februar verschärfte sie den Ton und warf der SPD Wählertäuschung vor: Die Sozialdemokraten würden nicht sagen, wie sie ihre Wahlversprechen finanzieren wollten. Insgesamt beliefen sich ihre Forderungen auf mehr als 250 Millionen im Jahr. Damit sei es unmöglich, den Kurs der Haushaltskonsolidierung fortzusetzen, was angesichts

20 Vgl. „Der Eigenwillige: Smart, aber herzlich“, in: kn-online.de vom 17. April 2012 (Abruf am 3. Mai 2012).

21 Siehe „Was Albig gleich nach der Wahl anpacken will“, in: ebenda vom 24. April 2012 (Abruf am 3. Mai 2012); „Wer will was? Die Ziele der Parteien“, in: Hamburger Abendblatt vom 2. Mai 2012, S. $4-5$.

22 Joachim Krause, zitiert in: „Die Wahl der Spitzenkräfte“, in: Hamburger Abendblatt vom 10. April 2012, S. 14.

23 Zitiert in: Frank Pergande, Kämpferisch und anders. Robert Habeck ist Spitzenkandidat der Grünen in Schleswig-Holstein, in: FAS vom 8. April 2012, S. 8.

24 Vgl. „Landtagswahl 2012. Themen“, in: sh.gruene.de (Abruf am 31. März 2012).

25 Siehe „Inhalte machen den Unterschied, Pressemitteilung vom 16. April 2012“, in: sh.gruene.de (Abruf am 23. Juli 2012); „Grüne stellen 100-Tage-Regierungsprogramm vor“, in: kn-online.de vom 16. April 2012 (Abruf am 3. Mai 2012). 
der in der Verfassung verankerten Schuldenbremse auf einen kalkulierten Verfassungsbruch hinauslaufe. ${ }^{26}$ Insgesamt versuchte die CDU sich als verlässlicher Garant haushalts- und wirtschaftspolitischer Solidität zu präsentieren: Sie versprach, bei den angekündigten Stellenkürzungen im Öffentlichen Dienst zu bleiben - die Polizei jedoch von den Kürzungen auszunehmen. In der Wirtschafts- und Infrastrukturpolitik befürwortete sie alle größeren Investitionsvorhaben im Straßenverkehrsbereich sowie den Ausbau der Flughäfen in Lübeck und auf Sylt. Ebenso wie die Opposition trat die CDU für eine Beschleunigung der Energiewende ein; in der Schulpolitik war sie ebenfalls nicht weit von der Opposition entfernt, bekannte sich aber eindeutiger als diese auch langfristig zum Erhalt des Gymnasiums. ${ }^{27}$ Nachdem auch das einzige TV-Duell vier Tage vor der Wahl keinen Umschwung zugunsten der CDU gebracht hatte, verließ sie jedoch ihren Sparkurs und kündigte bis zum Jahre 2020 Investitionen von 350 Millionen Euro in den Ausbau von Krippen- und Kita-Plätzen an. Finanziert werden sollte das Programm durch Einnahmen aus einem Steuerabkommen mit der Schweiz, für das es aber zum damaligen Zeitpunkt noch keine politische Mehrheit im Bundesrat gab. ${ }^{28}$

Anders als de Jager konnte FDP-Spitzenkandidat Kubicki seiner durchaus vorhandenen Neigung zum Populismus von Beginn an relativ freien Lauf lassen. Mit einer Regierungsbeteiligung der Liberalen rechnete ohnehin niemand - für die Partei ging es ums politische Überleben. Da mochte es dann vielleicht auch niemand verwundern, dass die FDP angesichts der Überschüsse der gesetzlichen Krankenkassen die Abschaffung der Praxisgebühr forderte, für eine Verdoppelung der Pendlerpauschale eintrat und sogar für die Einführung einer "Reichensteuer" - allerdings erst ab einem Jahreseinkommen von 250.000 Euro bei Alleinstehenden. ${ }^{29}$ Wie die CDU profilierte sich auch die FDP als Garant der Haushaltskonsolidierung und einer wachstumsfreundlichen Wirtschafts- und Verkehrspolitik. Stärker noch als diese stellte sie sich den Wählern als Verteidiger des Gymnasiums vor und befürwortete als einzige Partei in Schleswig-Holstein die Rückkehr zum neunjährigen Abitur. ${ }^{30}$ Insgesamt aber standen in der medialen Berichterstattung über den Wahlkampf der FDP weniger die politischen Inhalte im Zentrum als vielmehr die Person und die „Methode Kubicki“: Mit gezielten Provokationen gegen die Bundes-FDP kultivierte er sein Image als (Anti-)Politiker, der sagt, was er denkt. Damit - und mit einigen persönlichen Bekenntnissen - gelang es ihm wie keinem anderen Wahlkämpfer aus Schleswig-Holstein, auch bundesweit Fernseh- und Medienresonanz zu erzielen - bei Günther Jauch war er im März ebenso zu Gast wie bei Markus Lanz. ${ }^{31}$

26 Vgl. „CDU wirft SPD unverantwortliche Wahlversprechen vor“, in: kn-online.de vom 8. Februar 2012 (Abruf am 10. April 2012).

27 Siehe „Wer will was? Die Ziele der Parteien“, in: Hamburger Abendblatt vom 2. Mai 2012, S. 4-5.

28 Dazu „CDU-Wahlgeschenk in letzter Minute“, in: ebenda vom 4. Mai 2012, S. 14.

29 Vgl. „Gesundheitsminister Garg: Praxisgebühr abschaffen“, in: kn-online.de vom 14. Februar 2012 (Abruf am 10. April 2012); „Kubicki für höhere Reichensteuer und doppelte Pendlerpauschale“, in: ebenda vom 21. April 2012 (Abruf am 3. Mai 2012).

30 Siehe „Wer will was? Die Ziele der Parteien“, in: Hamburger Abendblatt vom 2. Mai 2012, S. 4- 5.

31 Vgl. Majid Sattar, Gegen die FDP für die FDP, in: faz.net vom 9. April 2012 (Abruf am 10. April 2012); Christiane Hoffmann / Wolfgang Kubicki, „Den haut nichts aus den Schlappen“, in: faz.net vom 28. April 2012 (Abruf am 2. Mai 2012); Bodo Stade, Die Liberalen setzen voll auf Wolfgang Kubicki: Er muss mal kurz die Welt retten, in: kn-online.de vom 17. April 2012 (Abruf am 3. Mai 2012). 
In gewisser Weise ebenfalls ein Medienphänomen, machten die Piraten weniger durch politische Inhalte als vielmehr durch persönliche Schrullen auf sich aufmerksam. Ihr Wahlprogramm war sehr lückenhaft und gewann vor allem durch den Hinweis eines Bloggers Prominenz, dass es in großen Teilen aus den Programmen anderer Landesverbände abgeschrieben sei. ${ }^{32}$ Bemerkenswert war auch die Tatsache, dass unter den ersten neun Kandidaten auf der Landesliste der Partei drei Mitglieder einer Familie zu finden waren: Spitzenkandidat Torge Schmidt, dessen Mutter Birgitt Piepgras (Platz neun) und ihr Ehemann Hans-Heinrich Piepgras (Platz sieben). ${ }^{33}$ Ansonsten sorgten vor allem die Kontroversen um den Umgang mit antisemitischen, rechtsextremen, sexistischen und manchmal auch nur unbedachten Äußerungen einzelner Parteimitglieder für Schlagzeilen. Hinzu traten Personaldiskussionen um den Berliner Landesvorstand und den neu zu wählenden Bundesvorstand auf dem Bundesparteitag in Neumünster Ende April. ${ }^{34}$

Von der Linkspartei gab es nicht viel zu vermelden: Sie glaubte angesichts der Konkurrenz der Piraten, ihrer mageren Erfolgsbilanz im Landtag und der Führungskrise in Berlin wohl selbst nicht daran, wieder in den Landtag einziehen zu können. „Links neben der Spur“35 wurde sie deshalb von den Medien weitgehend „links liegen gelassen“36 und lud allenfalls zum Texten anspielungsreicher Schlagzeilen ein. Etwas mehr Beachtung fand schließlich die Partei der dänischen Minderheit - nicht zuletzt, weil sie ein klares Koalitionssignal in Richtung Rot-Grün abgegeben hatte. Der SSW legte seinen Schwerpunkt wie immer auf die Bildungs-, Sozial- und Minderheitenpolitik. Er schrieb sich den Kampf für eine bessere Kinderbetreuung, eine vollständige staatliche Finanzierung der dänischen Schulen, eine verlässliche Hochschulpolitik und gegen Kürzungen im sozialen Bereich auf die Fahnen. ${ }^{37}$

\section{Das Wahlergebnis}

Das Wahlergebnis trat im Großen und Ganzen so ein, wie es die Umfragen vorausgesagt hatten: Die regierende Koalition wurde abgewählt. Für SPD und Grüne reichte es dennoch nicht zur Mehrheit; sie waren für die Regierungsbildung auf die Unterstützung des SSW angewiesen. Die Piraten zogen erstmals, die FDP erneut in den Kieler Landtag ein, während die 2009 erstmals gewählten Linken an der Sperrklausel scheiterten.

Die CDU blieb mit 30,8 Prozent der Zweitstimmen (minus 0,7 Prozentpunkte gegenüber 2009) knapp stärkste Partei vor der SPD (30,4 Prozent, plus 5,0 Punkte). Etwas deutlicher noch - 36,8 gegenüber 35,8 Prozent für die SPD - fiel der Vorsprung der Union bei

32 Siehe „Plagiatsvorwürfe beim Wahlprogramm“, in: faz.net vom 15. April 2012 (Abruf am 19. April 2012).

33 Vgl. „Schleswig-Holsteins Piraten in Familienhand“, in: shz.de vom 20. April 2012 (Abruf am 23. Juli 2012).

34 Hierzu zum Beispiel „Tolerant bis zur Schmerzgrenze“, in: faz.net vom 18. April 2012; „Piratenpartei uneins über Umgang mit Extremisten“, in: faz.net vom 18. April 2012 (Abruf am 19. April 2012); „Piraten wählen neuen Vorstand und zeigen Rechten die Rote Karte“, in: kn-online.de vom 29. April 2012 (Abruf am 3. Mai 2012).

35 Faz.net, 24. April 2012 (Abruf am 2. Mai 2012).

36 Hamburger Abendblatt vom 12. April 2012, S. 14.

37 Vgl. „SSW läutet in Schleswig heiße Phase des Wahlkampfs ein“, in: kn-online.de vom 31. März 2012 (Abruf am 10. April 2012). 
den Erststimmen aus, was ihr zwei Drittel der Direktmandate einbrachte. Alle 22 Abgeordneten der CDU zogen als Direktkandidaten in den Landtag ein. Die SPD gewann demgegenüber 13 Wahlkreise, die restlichen neun ihrer ebenfalls 22 Abgeordneten gelangten über die Liste in den Landtag. Drittstärkste Partei wurden die Grünen, die 13,2 Prozent der Zweitstimmen (plus 0,8 Punkte) erzielten. Sie sind im neuen Landtag mit zehn Abgeordneten vertreten. Als Wahlsieger konnten sich FDP und Piraten fühlen, die jeweils 8,2 Prozent der Stimmen erhielten - und das obwohl die FDP gegenüber der Landtagswahl 2009 6,7 Punkte verlor. Nachdem sie jedoch zwischenzeitlich in den Wahlumfragen unter fünf Prozent gefallen war, wurde das Ergebnis zu Recht als Erfolg der Partei und ihres Vorsitzenden Kubicki gewertet. Liberale wie Piraten sind im neuen Landtag mit jeweils sechs Mandaten vertreten. Der SSW konnte sich leicht auf 4,6 Prozent (plus 0,3 Punkte) verbessern. Da er als politische Vertretung der dänischen Minderheit von der Sperrklausel befreit ist, entsendet er drei Abgeordnete ins Parlament, das wie 2009 aus sechs Parteien besteht. Die Linke scheiterte erwartungsgemäß mit 2,3 Prozent der Stimmen (minus 3,7 Punkte) an der Sperrklausel (vgl. Tabelle 1).

Beim Vergleich mit dem Landtagswahlergebnis von vor drei Jahren ist zu beachten, dass das neue Wahlrecht das Entstehen von Überhang- und Ausgleichmandaten diesmal verhinderte. Dem neuen 18. Landtag gehören deshalb wie vorgesehen nur 69 Abgeordnete an, während der 17. sich noch aus 95 Volksvertretern zusammensetzte. Vergleiche machen folglich wenig Sinn; faktisch verloren alle schon im 17. Landtag vertretenen Parteien an Mandaten - besonders schmerzhaft CDU (minus zwölf) und FDP (minus acht), aber auch SPD (minus drei), Grüne (minus zwei) und SSW (minus ein Mandat). Ebenso wenig sinnvoll ist ein Vergleich der Wahlbeteiligung mit 2009, weil damals die Landtagswahl am selben Tag wie die Bundestagswahl stattfand. Auch gegenüber der Landtagswahl 2005 ging die Wahlbeteiligung jedoch immer noch um 6,4 Punkte auf 60,1 Prozent zurück (vgl. Tabelle 1).

\subsection{Wahlmotive: Themen, Kandidaten, Kompetenzen und Koalitionen}

Noch stärker als 2009, als zeitgleich der Bundestag gewählt wurde, dominierte diesmal die Landespolitik die Entscheidungen der Wähler: Knapp zwei von drei gaben an, dass landespolitische Themen für sie ausschlaggebender gewesen seien als bundespolitische Fragen; bei knapp einem Drittel war es umgekehrt. Die Wähler der Piratenpartei waren die einzigen, bei denen bundespolitische Wahlmotive mit 56 Prozent die landespolitischen (35 Prozent) überwogen. Der SSW dagegen wurde - nicht weiter verwunderlich - mit Abstand am stärksten, nämlich zu 85 Prozent, aus landespolitischen Erwägungen heraus gewählt. ${ }^{38}$

Generell flossen in die vielfältigen Motive der Wählerschaft Sachfragen zu einem größeren Teil ein als die Bewertung der Kandidaten und die Parteibindung: Für knapp die Hälfte der Wähler waren Sachfragen bei ihrer Entscheidung am wichtigsten, für jeweils ein Viertel die Kandidaten und die Parteibindung. Dabei gab es signifikante Unterschiede zwischen den Parteien und auch interessante Entwicklungen im diachronen Vergleich: Bei der SPD (34 Prozent, plus 14 Punkte gegenüber 2009) und der FDP (31 Prozent, plus zwölf Punkte)

38 Siehe Forschungsgruppe Wahlen, a.a.O. (Fn. 6), S. 26. 
erreichte der Anteil der Personenwähler die höchsten Werte; bei den Piraten und den Linken spielte die Kandidatenfrage dagegen so gut wie keine Rolle. Auch die Grünen und der SSW wurden weit überwiegend aus inhaltlichen Gründen gewählt; immerhin aber gelang vor allem Habeck eine deutliche Steigerung der Personenwähler auf 15 Prozent (gegenüber nur acht Prozent 2009). Die CDU war diejenige Partei, die vergleichsweise am stärksten aus Parteiloyalität gewählt wurde. Für knapp ein Drittel ihrer Wähler stand die Parteibindung bei der Wahlentscheidung im Vordergrund. Damit einher geht der Befund, dass sie am stärksten (zu 70 Prozent) aus Überzeugung gewählt wurde; am wenigsten (zu 32 Prozent)

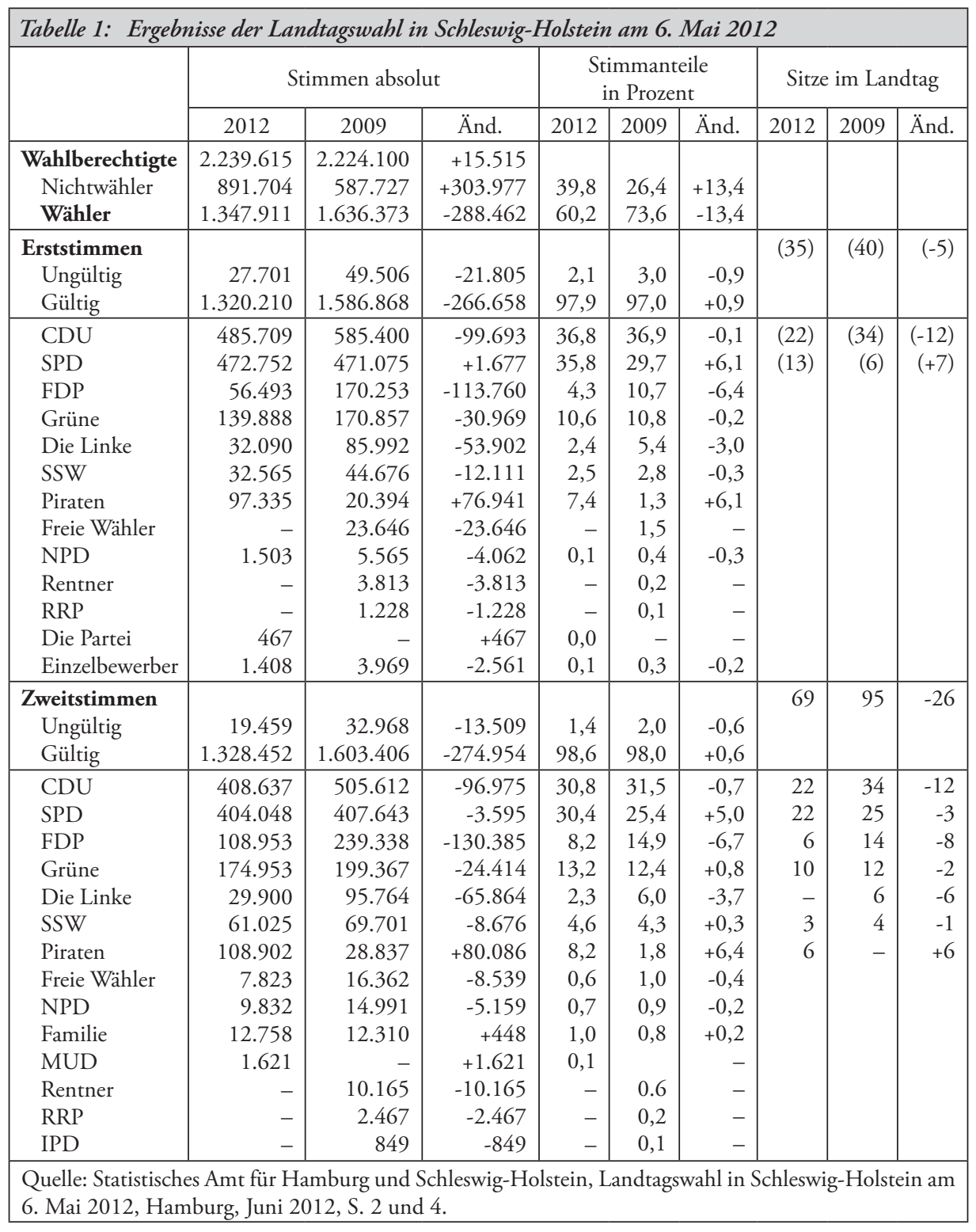


wurden dies die Piraten. Zwei Drittel ihrer Wähler gaben an, dass sie von anderen Parteien enttäuscht waren. ${ }^{39}$

Die Antworten auf die Frage nach den wichtigsten Themenfeldern im Wahlkampf hängen bekanntlich auch sehr von der Fragestellung und der Fragekonzeption (offen oder geschlossen) ab: Die Forschungsgruppe Wahlen ermittelte in ihrer Vorwahlerhebung zwei bis sechs Tage vor der Wahl - mit einer offenen Frage ohne vorgegebene Antwortmöglichkeiten - das Themenfeld Schule und Bildung als das mit Abstand wichtigste Problem, das von 41 Prozent der Wähler genannt wurde. Die Themen Arbeitsplätze und Verschuldung folgten auf dem zweiten und dritten Platz mit jeweils 24 Prozent der Nennungen, während jeweils elf Prozent Probleme aus den Themenfeldern Verkehr und Umwelt/Klima/Energie nannten. ${ }^{40}$ Infratest dimap kam mit seiner Wahltagbefragung und zwölf vorgegebenen Antwortmöglichkeiten zu etwas anderen Ergebnissen: Hier wurde die Wirtschaftspolitik am häufigsten genannt, gefolgt von Schule/Bildung, sozialer Gerechtigkeit, Arbeitsmarkt und Umwelt/Klima. Natürlich gab es auch hier Unterschiede zwischen den Anhängern verschiedener Parteien: Den Wählern von CDU und FDP waren wirtschaftspolitische Themen am wichtigsten, während Linkspartei, SPD und Piraten vorrangig aufgrund sozialpolitischer Themen gewählt wurden. Die Grünen punkteten am stärksten mit umweltpolitischen, der SSW mit bildungspolitischen Themen. Die Bedeutung der Bildungspolitik zeigte sich vor allem darin, dass sie bei Wählern aller Parteien eine annähernd gleichmäßig verteilte Priorität genoss (vgl. Tabelle 2).

\begin{tabular}{|l|c|c|c|c|c|c|c|c|}
\hline Tabelle 2: & $\begin{array}{c}\text { Die fünf wichtigsten Themen der Wablentscheidung insgesamt und bezogen auf die } \\
\text { Wähler der einzelnen Parteien, Prozentwerte (Spitzenwert jeweils fett markiert) }\end{array}$ \\
\hline Thema & Insgesamt & CDU & SPD & FDP & Grüne & Linke & SSW & Piraten \\
\hline 1. Wirtschaftspolitik & $\mathbf{3 6}$ & $\mathbf{5 8}$ & 27 & $\mathbf{5 6}$ & 17 & 28 & 15 & 20 \\
2. Schule/Bildung & 32 & 26 & 33 & 35 & 37 & 36 & $\mathbf{4 7}$ & 25 \\
3. Soziale Gerechtigkeit & 30 & 12 & $\mathbf{4 1}$ & 14 & 41 & $\mathbf{7 0}$ & 41 & $\mathbf{3 4}$ \\
4. Arbeitsmarkt & 26 & 26 & 31 & 22 & 18 & 38 & 22 & 21 \\
5. Umwelt/Klima & 19 & 9 & 15 & 6 & $\mathbf{6 1}$ & 20 & 20 & 10 \\
\hline
\end{tabular}

Frage: Welcher der folgenden Aspekte war für Ihre Wahlentscheidung letztlich ausschlaggebend? (Bis zu zwei Nennungen möglich).

Quelle: Infratest dimap, WahlREPORT, a.a.O. (Fn. 39), S. 46.

Die den Parteien zugewiesenen Kompetenzen gingen im Wesentlichen mit den Themenprioritäten der Parteianhänger konform. Allerdings fielen die Kompetenzwerte für FDP, SSW und Piraten auf allen Politikfeldern mit maximal drei Prozent so niedrig aus, dass sie im Grunde noch im statistischen Fehlerbereich lagen. Der Linkspartei ging es im Prinzip ähnlich, immerhin aber wurde ihr in der Sozialpolitik noch von sechs Prozent der Wähler eine Lösungskompetenz zugeschrieben, ohne dass ihr dies jedoch etwas genutzt hätte. Der CDU wurde am meisten zugetraut mit Blick auf die Arbeitsplatzsicherung, die Wirtschaftspolitik und den Abbau der Verschuldung. In der Bildungs-, der Sozial- und der Familienpolitik lag demgegenüber die SPD vorne. Die Grünen distanzierten auf den Feldern der Umwelt- und der Energiepolitik CDU und SPD mit deutlichem Abstand. Hinsichtlich des generellen

39 Vgl. Infratest dimap, WahlREPORT, Landtagswahl Schleswig-Holstein 2012. Eine Analyse der Wahl vom 6. Mai 2012, Berlin 2012, S. 44 - 45; Patrick Horst, a.a.O. (Fn. 3), S. 383 - 384.

40 Siehe Forschungsgruppe Wahlen, a.a.O. (Fn. 6), S. 26. 
Zutrauens, die wichtigsten Probleme in der Zukunft lösen zu können, lag die SPD mit fünf Prozentpunkten vor der Union (36 gegenüber 31 Prozent). ${ }^{41}$

In der bundesdeutschen Koalitionsdemokratie ${ }^{42}$ entscheiden Wähler nicht nur aufgrund ihrer Kandidaten-, Themen- und Parteieinschätzungen, sondern auch aufgrund ihrer Koalitionspräferenzen. Unmittelbar vor der Wahl wurde ein rot-grünes Bündnis von den Wählern am besten bewertet, gefolgt von der „Dänen-Ampel“ und einer Großen Koalition. ${ }^{43}$ Strategisches „Lager“- und Koalitionswahlverhalten lässt sich bis zu einem gewissen Grade auch am Stimmensplitting ablesen. Wie schon 2009 machten FDP-Wähler davon am ausgiebigsten Gebrauch: Diesmal gaben sogar 54 Prozent der FDP-Zweitstimmenwähler ihre Erststimme einem Kandidaten der CDU, was zu deren Gewinn von zwei Dritteln aller Direktmandate beitrug. Deutlich häufiger als 2009 votierten 2012 auch die Grünen-Wähler mit ihrer Erststimme für die SPD: 37 anstatt vormals 27 Prozent; und auch knapp ein Drittel der SSW-Wähler splittete zugunsten der SPD. Die im Wahlkampf angekündigte und später auch gebildete „Dänen-Ampel“ spiegelte sich also im konkreten Wahlverhalten erkennbar wider. ${ }^{44}$

\subsection{Sozialstrukturelle und regionale Bestimmungsfaktoren des Wahlverhaltens}

Bei der Landtagswahl in Schleswig-Holstein waren die beiden vormaligen Volksparteien CDU und SPD (sowie die FDP) von der sozialstrukturellen Zusammensetzung ihrer Wählerschaft her eindeutig die ältesten Parteien. Gemessen an einem 35-Prozent-Kriterium ${ }^{45}$, das beide Parteien in der Gesamtwählerschaft nun schon zum zweiten Mal nach 2009 verfehlt haben, konnten sich CDU und SPD ihren Volksparteienstatus allein noch in der Gruppe der über 60-jährigen Wähler bewahren: Vor allem die über 60-jährigen Frauen wählten zu drei Vierteln CDU und SPD; in dieser Gruppe waren die Zugewinne der SPD zudem am stärksten (vgl. Tabelle 3). Demgegenüber brachten es die beiden großen Parteien in der jüngsten Gruppe der 18- bis 29-Jährigen zusammen nur auf weniger als die Hälfte der Wähler. Die Piraten zogen 20 Prozent dieser Gruppe, die Grünen 16 Prozent an (vgl. Tabelle 3). Umgerechnet auf die gesamte Wählerschaft der Parteien machten die 18- bis 29-Jährigen 27 Prozent bei den Piraten aus, aber nur 14 Prozent bei den Grünen, die somit den Status der ,jüngsten “ Partei an die Piraten abgeben mussten. ${ }^{46}$ Allerdings relativiert sich die Betrachtung bei einer Berücksichtigung der Geschlechter: Während 24 Prozent der 18bis 29-jährigen Männer die Piraten wählten (genauso viele wie die CDU und etwas mehr als die SPD), taten dies nur 15 Prozent der jungen Frauen. Bei den Grünen war es umge-

41 Vgl. Infratest dimap, a.a.O. (Fn. 39), S. 26 - 27.

42 Siehe jetzt Patrick Horst, Die deutsche Koalitionsdemokratie - eine empirische Analyse, in: Frank Decker / Eckhard Jesse (Hrsg.), Die deutsche Koalitionsdemokratie vor der Bundestagswahl 2013, Baden-Baden 2012, im Erscheinen.

43 Vgl. Forschungsgruppe Wahlen, a.a.O. (Fn. 6), S. 19; Infratest dimap, a.a.O. (Fn. 39), S. 38.

44 Siehe Infratest dimap, a.a.O. (Fn. 39), S. $46-47$.

45 Vgl. Peter Lösche, Ende der Volksparteien, in: APuZ, B 51/2009, S. 7, hat drei quantitative Kriterien für eine Volkspartei definiert: Nur wenn dauerhaft mindestens 35 Prozent der Wahlberechtigten eine Partei wählen, ein Prozent ihr als Mitglieder und 0,1 Prozent als Funktionäre zur Verfügung stehen, kann sie den Anspruch, eine Volkspartei, das heißt eine Massenwähler-, Mitglieder- und Funktionärspartei zu sein, auch erfüllen.

46 Vgl. Forschungsgruppe Wahlen, a.a.O. (Fn. 6), S. 67. 
kehrt: Sie wurden von 20 Prozent der 18- bis 29-jährigen Frauen, aber nur von 13 Prozent der jungen Männer gewählt (vgl. Tabelle 3). Dieser Geschlechterbias hielt sich durch alle Altersgruppen hindurch, so dass die Grünen zu 60 Prozent von Frauen, die Piraten zu 60 Prozent von Männern gewählt wurden. In allen anderen Parteien war die Geschlechterverteilung unter den Wählern relativ ausgewogen, nur die FDP verzeichnete in ihrer Wählerschaft einen signifikanten Männerüberhang von $56 \mathrm{zu} 44$ Prozent. ${ }^{47}$

Angesichts der Altersstruktur der Wählerschaft konnte es nicht überraschen, dass unter den Wählern von CDU, SPD und FDP die Rentner stark überproportional vertreten waren. Die Berufstätigen waren demgegenüber bei allen drei Parteien leicht, die Arbeitslosen bei CDU und FDP stark unterrepräsentiert. Grüne und Piraten erzielten leicht überproportionale Ergebnisse bei den Berufstätigen; die Piraten zogen überdies knapp ein Viertel der Arbeitslosen an. In dieser Wählergruppe gewannen sie gegenüber 200920 Prozentpunkte hinzu und wurden nur noch von der SPD übertroffen, die 28 Prozent der Arbeitslosen wählten. Unter den Berufsgruppen zeigte sich im Wesentlichen die altbekannte Verteilung: Arbeiter wählten überdurchschnittlich häufig die SPD (und den SSW sowie die Piraten); Beamte und Selbständige sahen sich am besten repräsentiert durch CDU, FDP und Grüne; die Landwirte wählten extrem überproportional CDU und extrem unterdurchschnittlich alle anderen Parteien mit Ausnahme der FDP. Die mit Abstand größte Berufsgruppe der Angestellten, bei der 2009 noch die CDU mit 31 Prozent die Nase vorn hatte, wählte diesmal zu 32 Prozent die SPD (vgl. Tabelle 3).

Ähnlich beständig wie unter den Berufsgruppen war auch das Wahlverhalten mit Blick auf die Merkmale Konfession und Bildung: 60 Prozent der schleswig-holsteinischen Bevölkerung sind Protestanten, nur sechs Prozent Katholiken, und 28 Prozent sind konfessionslos. ${ }^{48}$ Die CDU war wie schon 2009 auch 2012 wieder stärkste Partei unter beiden Konfessionsgruppen, während die SPD bei den Konfessionslosen vorne lag. Konfessionslose wählten auch überdurchschnittlich häufig Grüne und Piraten. In den drei Parteien der „Dänen-Ampel“ sind die Protestanten und die Konfessionslosen überrepräsentiert; die katholische Minderheit sieht sich von CDU und FDP besser vertreten. In den Bildungsgruppen erzielte die SPD ihre besten Ergebnisse unter Wählern mit Hauptschulabschluss, während die Grünen und die FDP bei Hochschulabsolventen besonders gut abschnitten. Grüne und Piraten zogen überdurchschnittlich Wähler mit Abitur an, CDU und SSW hatten ihre Spitzenwerte bei Wählern mit mittlerer Reife und Hauptschulabschluss. Wollte man eine Rangliste nach dem formalen Bildungsgrad der Wähler erstellen, so sähe sie wie folgt aus: Grüne vor FDP, Piraten, CDU, SSW und SPD (vgl. Tabelle 3).

Die traditionellen Hochburgen der Parteien spiegelten sich auch 2012 im Wahlverhalten der Schleswig-Holsteiner wider - wenn auch teils auf niedrigerem Niveau: Der SSW erreichte im nördlichen Landesteil Schleswig fast elf Prozent und wurde hier viertstärkste Partei; in den Wahlkreisen Flensburg, Flensburg-Land, Schleswig-Nord und Südtondern wurde er sogar dritte Kraft. Im Landesteil Holstein erreichte der SSW dagegen nur knapp drei Prozent. Die CDU war neben dem SSW die einzige Partei, die im ländlich geprägten Landesteil Schleswig stärker abschnitt als im Landesdurchschnitt (vgl. Tabelle 3). In Holstein hatte sie ihre Hochburgen im Westen (beide Dithmarscher Wahlkreise und Steinburg-West), in Segeberg-Ost (Ostholstein) und in Pinneberg-Nord (Hamburger Umland). In den größeren

47 Siehe ebenda.

48 Vgl. ebenda, S. 39. 


\begin{tabular}{|c|c|c|c|c|c|c|c|c|}
\hline \multicolumn{9}{|c|}{$\begin{array}{l}\text { Tabelle 3: Wablentscheidung in sozialen Gruppen und Räumen, Zeilen-Prozentwerte } \\
\text { (in Klammern Differenz in Prozentpunkten zu 2009) }\end{array}$} \\
\hline $\begin{array}{l}\text { Zweitstimmen- } \\
\text { ergebnis } 2012\end{array}$ & $\begin{array}{r}\text { CDU } \\
30,8 \\
(-0,7) \\
\end{array}$ & $\begin{array}{r}\text { SPD } \\
30,4 \\
(+5,0) \\
\end{array}$ & $\begin{array}{r}\text { FDP } \\
8,2 \\
(-6,7) \\
\end{array}$ & $\begin{array}{r}\text { Grüne } \\
13,2 \\
(+0,8) \\
\end{array}$ & \begin{tabular}{|r|} 
Linke \\
2,2 \\
$(-3,8)$ \\
\end{tabular} & $\begin{array}{r}\text { SSW } \\
4,6 \\
(+0,3) \\
\end{array}$ & \begin{tabular}{|r|} 
Piraten \\
8,2 \\
$(+6,4)$ \\
\end{tabular} & $\begin{array}{r}\text { Sonstige } \\
2,4 \\
(-1,3) \\
\end{array}$ \\
\hline $\begin{array}{l}\text { Geschlecht } \\
\text { Männlich } \\
\text { Weiblich }\end{array}$ & $\begin{array}{r}31(0) \\
30(-3)\end{array}$ & $\begin{array}{l}29(+4) \\
31(+6) \\
\end{array}$ & $\begin{array}{l}9(-8) \\
7(-7)\end{array}$ & $\begin{array}{r}11(0) \\
16(+2) \\
\end{array}$ & $\begin{array}{l}2(-4) \\
2(-3)\end{array}$ & $\begin{array}{r}5(0) \\
4(+1) \\
\end{array}$ & $\begin{array}{r}10(+8) \\
7(+6) \\
\end{array}$ & $\begin{array}{l}3(-1) \\
2(-1)\end{array}$ \\
\hline $\begin{array}{l}\text { Alter } \\
\text { 18-29 Jahre } \\
\text { 30-44 Jahre } \\
\text { 45-59 Jahre } \\
\text { Ab 60 Jahre } \\
\end{array}$ & $\begin{array}{l}22(-3) \\
30(+1) \\
26(+2) \\
38(-4) \\
\end{array}$ & $\begin{array}{l}24(+4) \\
24(+3) \\
31(+3) \\
35(+7) \\
\end{array}$ & $\begin{array}{r}5(-11) \\
6(-11) \\
7(-7) \\
11(-3) \\
\end{array}$ & $\begin{array}{r}16(+2) \\
17(+2) \\
17(+1) \\
7(+1) \\
\end{array}$ & $\begin{array}{l}3(-5) \\
3(-5) \\
3(-5) \\
1(-2)\end{array}$ & $\begin{array}{r}4(+1) \\
5(+1) \\
5(0) \\
4(0) \\
\end{array}$ & $\begin{array}{r}20(+11) \\
11(+9) \\
9(+8) \\
3(+3) \\
\end{array}$ & $\begin{array}{r}5(0) \\
5(0) \\
2(-2) \\
1(-2) \\
\end{array}$ \\
\hline $\begin{array}{l}\text { Alter und Geschlecht } \\
\text { 18-29 Jahre Mann } \\
\text { 18-29 Jahre Frau } \\
\text { 30-44 Jahre Mann } \\
\text { 30-44 Jahre Frau } \\
\text { 45-59 Jahre Mann } \\
\text { 45-59 Jahre Frau } \\
\text { Ab 60 Jahre Mann } \\
\text { Ab 60 Jahre Frau } \\
\end{array}$ & $\begin{array}{r}24(0) \\
20(-6) \\
31(+1) \\
28(-1) \\
27(+4) \\
26(0) \\
37(-3) \\
39(-7) \\
\end{array}$ & $\begin{array}{r}22(+3) \\
27(+6) \\
23(+2) \\
26(+4) \\
31(+3) \\
31(+3) \\
33(+5) \\
36(+10) \\
\end{array}$ & $\begin{array}{r}6(-11) \\
5(-11) \\
7(-12) \\
5(-10) \\
7(-8) \\
6(-6) \\
13(-3) \\
10(-4) \\
\end{array}$ & $\begin{array}{r}13(+1) \\
20(+3) \\
12(0) \\
21(+4) \\
15(0) \\
21(+1) \\
6(0) \\
9(+1) \\
\end{array}$ & & $\begin{array}{r}4(0) \\
4(+2) \\
5(+2) \\
5(0) \\
6(-1) \\
5(+1) \\
4(0) \\
4(0) \\
\end{array}$ & $\begin{array}{r}24(+14) \\
15(+9) \\
15(+12) \\
9(+8) \\
9(+8) \\
8(+8) \\
4(+4) \\
2(+2) \\
\end{array}$ & $\begin{array}{l}6(0) \\
5(0) \\
4(0) \\
5(0) \\
2(-1) \\
1(-2) \\
1(-2) \\
1(-1) \\
\end{array}$ \\
\hline $\begin{array}{l}\text { Erwerbsstatus } \\
\text { Berufstätige } \\
\text { Rentner } \\
\text { Arbeitslose } \\
\end{array}$ & $\begin{array}{l}27(-1) \\
38(-2) \\
17(+2) \\
\end{array}$ & $\begin{array}{l}29(+5) \\
35(+6) \\
28(+7) \\
\end{array}$ & & $\begin{array}{r}16(+1) \\
7(+1) \\
13(0) \\
\end{array}$ & $\begin{array}{r}3(-4) \\
1(-2) \\
8(-11) \\
\end{array}$ & $\begin{array}{r}5(0) \\
4(0) \\
6(-4) \\
\end{array}$ & $\begin{array}{r}10(+8) \\
3(+3) \\
23(+20) \\
\end{array}$ & $\begin{array}{l}3(-1) \\
1(-2) \\
3(-5) \\
\end{array}$ \\
\hline $\begin{array}{l}\text { Berufsgruppe } \\
\text { Arbeiter } \\
\text { Angestellte } \\
\text { Beamte } \\
\text { Selbständige } \\
\text { Landwirte }\end{array}$ & $\begin{array}{r}25(0) \\
28(-3) \\
34(0) \\
38(-2) \\
80(+1)\end{array}$ & $\begin{array}{r}37(+6) \\
32(+6) \\
28(+7) \\
19(+3) \\
5(+2)\end{array}$ & $\begin{aligned} 5 & (-8) \\
8 & (-6) \\
11 & (-7) \\
14 & (-6) \\
7 & (-7)\end{aligned}$ & $\begin{array}{r}8(-1) \\
15(+1) \\
16(-1) \\
15(+3) \\
3(+2)\end{array}$ & $\begin{array}{l}3(-7) \\
2(-3) \\
1(-2) \\
2(-2) \\
-(-2)\end{array}$ & $\begin{array}{r}6(0) \\
5(+1) \\
5(0) \\
2(-1) \\
2(+2)\end{array}$ & $\begin{array}{r}13(+11) \\
8(+6) \\
3(+2) \\
8(+7) \\
2(+2)\end{array}$ & $\begin{array}{r}4(-2) \\
2(-1) \\
2(+1) \\
1(-3) \\
-(-1)\end{array}$ \\
\hline $\begin{array}{l}\text { Konfession } \\
\text { Katholisch } \\
\text { Evangelisch } \\
\text { Keine } \\
\end{array}$ & $\begin{array}{r}41(+5) \\
35(0) \\
23(-1) \\
\end{array}$ & $\begin{array}{l}25(+3) \\
31(+5) \\
29(+4) \\
\end{array}$ & $\begin{array}{r}10(-6) \\
8(-7) \\
8(-8) \\
\end{array}$ & $\begin{array}{r}11(0) \\
12(+1) \\
16(+1) \\
\end{array}$ & $\begin{array}{l}2(-4) \\
1(-3) \\
4(-6)\end{array}$ & $\begin{array}{l}2(-1) \\
5(+1) \\
5(+1)\end{array}$ & $\begin{array}{r}8(+5) \\
7(+5) \\
12(+9) \\
\end{array}$ & $\begin{array}{l}1(-2) \\
2(-2) \\
4(-1)\end{array}$ \\
\hline $\begin{array}{l}\text { Schulbildung } \\
\text { Hauptschulabschluss } \\
\text { Mittlere Reife } \\
\text { Hochschulreife } \\
\text { Hochschulabschluss }\end{array}$ & $\begin{array}{r}32(+3) \\
33(0) \\
30(0) \\
28(-2) \\
\end{array}$ & $\begin{array}{l}39(+7) \\
29(+6) \\
24(+2) \\
24(+4)\end{array}$ & $\begin{array}{r}7(-7) \\
8(-8) \\
7(-7) \\
11(-6)\end{array}$ & $\begin{array}{r}5(-2) \\
11(+1) \\
19(0) \\
23(+3)\end{array}$ & $\begin{array}{l}2(-5) \\
2(-4) \\
3(-2) \\
2(-3) \\
\end{array}$ & $\begin{array}{c}6(+2) \\
5(0) \\
4(0) \\
3(0)\end{array}$ & $\begin{array}{r}7(+6) \\
9(+8) \\
10(+7) \\
7(+4) \\
\end{array}$ & $\begin{array}{r}2(-3) \\
3(-1) \\
3(0) \\
2(+1)\end{array}$ \\
\hline $\begin{array}{l}\text { Region } \\
\text { Schleswig } \\
\text { Holstein } \\
\text { Kiel } \\
\text { West-Holstein } \\
\text { Ost-Holstein } \\
\text { Hamburger Umland } \\
\end{array}$ & $\begin{aligned} & 32(-1) \\
& 30(-1) \\
& 20(-2) \\
& 34(0) \\
& 30(-1) \\
& 32(-1)\end{aligned}$ & $\begin{array}{l}26(+4) \\
32(+5) \\
36(+6) \\
29(+5) \\
32(+5) \\
31(+5)\end{array}$ & $\begin{array}{l}8(-6) \\
8(-7) \\
7(-6) \\
9(-8) \\
8(-7) \\
9(-6)\end{array}$ & $\begin{array}{r}12(+1) \\
14(+1) \\
18(+1) \\
12(+1) \\
13(+1) \\
13(0)\end{array}$ & $\begin{array}{l}2(-3) \\
2(-4) \\
4(-4) \\
2(-4) \\
2(-4) \\
2(-4)\end{array}$ & $\begin{array}{r}11(0) \\
3(0) \\
4(-1) \\
3(0) \\
3(0) \\
2(+1) \\
\end{array}$ & $\begin{array}{l}7(+6) \\
8(+7) \\
9(+6) \\
9(+7) \\
8(+7) \\
8(+6)\end{array}$ & $\begin{array}{l}2(-1) \\
3(-1) \\
2(-1) \\
3(-2) \\
3(-1) \\
2(-1)\end{array}$ \\
\hline
\end{tabular}


Städten war die CDU unverändert schwach. Dort erzielte die SPD ihre besten Ergebnisse: allen voran in der traditionsreichen SPD-Hochburg Kiel Ost, gefolgt von den beiden Lübecker Wahlkreisen, Kiel Nord und West sowie Ostholstein-Nord. Im Landesteil Schleswig schnitt die SPD wie gewohnt unterdurchschnittlich ab (vgl. Tabelle 3), hier konnte sie nur im Wahlkreis Flensburg ein Direktmandat erobern. Die FDP war am stärksten im Hamburger Umland (Raum Stormarn, Pinneberg Nord), in Ostholstein Süd, in Segeberg West und im Raum Dithmarschen präsent; die Grünen erzielten ihre besten Wahlkreisergebnisse in Kiel (Nord und West), im Westen Lübecks, in der Region Stormarn im Hamburger Umland und in Flensburg; die Piraten wurden überdurchschnittlich häufig in Kiel (Ost und West) sowie in den beiden Lübecker und Segeberger Wahlkreisen gewählt. ${ }^{49}$

\section{Regierungsbildung und Formierung der Opposition}

Die einzige Gewissheit am Wahlabend war, dass die schwarz-gelbe Koalition abgewählt war. CDU-Spitzenkandidat de Jager hielt angesichts des knappen Vorsprungs vor der SPD zunächst noch die Illusion aufrecht, ein Regierungsbündnis schmieden zu können und bot „allen infrage kommenden Parteien“ Gespräche an. ${ }^{50}$ SPD, Grüne und SSW machten jedoch schon am Wahlabend deutlich, dass sie eine „Dänen-Ampel“ bilden wollten, auch wenn sie nur über eine Stimme Mehrheit im Landtag verfügten - die sie nun ihrerseits pikanterweise einer Neuerung im Wahlrecht verdankten, nämlich der von den Grünen und dem SSW durchgesetzten Umstellung des Auszählverfahrens von d'Hondt auf Sainte Laguël Schepers. ${ }^{51}$ Bereits am Tag nach der Wahl legten sie sich auf einen Fahrplan zur Koalitionsbildung fest, der auch eingehalten wurde. ${ }^{52}$

SPD und Grüne nahmen ihre Gespräche am Donnerstag nach der Wahl auf. Am Freitag verhandelten die beiden Parteien jeweils getrennt mit dem SSW; am Montag trafen sich alle drei Parteien zu abschließenden Sondierungen. Nachdem die Parteigremien der Aufnahme von Koalitionsverhandlungen zugestimmt hatten, begannen diese zehn Tage nach der Wahl am Mittwoch, den 16. Mai. Die Gespräche wurden in einer Großen Verhandlungsrunde geführt, der jeweils zwölf Vertreter der drei Koalitionspartner angehörten. Formale Verhandlungsführer waren die Parteivorsitzenden Ralf Stegner (SPD), Eka von Kalben (Grüne) und Flemming Mayer (SSW), faktisch waren Albig sowie die beiden Fraktionsvorsitzenden Habeck und Spoorendonk von kaum minderer oder größerer Bedeutung. Dieser Runde arbeiteten sechs Facharbeitsgruppen zu, in der jeweils drei Politiker jeder Partei saßen. Die strittigen Punkte im Bereich Finanzen, Personal und Ressortzuschnitt wurden von einer zehnköpfigen kleinen Verhandlungsgruppe der Parteispitzen aus jeweils drei Vertreten von SPD und SSW sowie vier Grünen geklärt. Nach zweieinhalb Wochen, am 3. Juni, war der Koalitionsvertrag fertig verhandelt. Er wurde auf Sonderparteitagen der drei Partner am 9. Juni gebilligt. Am 12. Juni wählte der Landtag Albig mit den Stimmen der Koalition und von wahrscheinlich zwei Piraten zum Ministerpräsidenten, am folgenden Tag wurden seine Minister vom Land-

49 Siehe ebenda, S. 75 - 85; Infratest dimap, a.a.O. (Fn. 39), S. 49 - 52.

50 Zitiert in: „Kopf an Kopf“, in: Hamburger Abendblatt vom 7. Mai 2012, S. 3.

51 Nach dem alten Verfahren hätte die Union anstelle der Grünen das letzte Mandat zugeteilt bekommen, vgl. „Neue Chance in einem Jahr“, in: Hamburger Abendblatt vom 11. Mai 2012, S. 16.

52 Vgl. „Eine Stimme mehr: Albig will mit Dänen regieren“, in: ebenda vom 8. Mai 2012, S. 1. 
tagspräsidenten vereidigt. Insgesamt hatte die Regierungsbildung 38 Tage in Anspruch genommen - was angesichts einer Koalition aus drei Partnern und eines neuen Koalitionstyps als zügig gelten kann. ${ }^{53}$

Die drei Parteien lagen inhaltlich dicht beieinander, sind mithin als „minimal range“ beziehungsweise „minimal connected winning coalition“ "zu bezeichnen. ${ }^{54}$ Einer relativ reibungslosen Koalitionsbildung sollten demnach keine unüberwindlichen Hürden im Wege stehen, dennoch gab es in Zeiten der Finanznot inhaltliche Differenzen zu klären: Die Verschuldungsbremse erfordert einen Abbau des strukturellen Haushaltsdefizits um 112 Millionen Euro pro Jahr bis 2020; gleichzeitig hatten aber vor allem SPD und SSW teure Wahlversprechen gemacht, ohne konkret realisierbare Finanzierungsvorschläge zu unterbreiten. Die Wahlversprechen der Grünen waren noch am billigsten, auch zeigten sie sich am stärksten von allen drei Partnern an der Fortführung des Sparkurses interessiert. Bei den beiden großen Verkehrsinfrastrukturprojekten (Autobahn A 20, Fehmarnbelt-Tunnel) traten die Grünen als Gegner auf, während die SPD beide Verkehrsprojekte und der SSW die A 20 befürwortete. Am geringsten waren die Meinungsunterschiede in der Bildungs-, Arbeitsmarkt- und Innenpolitik. ${ }^{55}$

Die Interessenverbände der mittelständischen Wirtschaft, die Industrie- und Handelskammer Schleswig-Holstein und die Unternehmensverbände Nord versuchten, auf den Prozess der Koalitionsbildung öffentlich Druck auszuüben, indem sie ihrer Furcht vor einer instabilen Dreier-Koalition Ausdruck verliehen. Insbesondere forderten sie eine konsequente Fortführung des Schuldenabbaus, eine investitionsfreundliche Politik und den zügigen Weiterbau der großen Verkehrsprojekte. ${ }^{56}$ Ihr Gang an die Öffentlichkeit war wohl der Tatsache geschuldet, dass sie ihren Einfluss auf die Unterhändler der Koalition schwinden sahen. Die öffentlich kaum vernehmbaren Interessenverbände der Windenergie, der Bio-Landwirtschaft, des Umweltschutzes, der Kommunen und der (Lehrer-)Gewerkschaften sollten sich im Ergebnis der Verhandlungen am Ende besser wiederfinden als die Wirtschaftsverbände.

Der fertige Koalitionsvertrag, der „neue Horizonte“ für das Land in Aussicht stellte, warb vor allem mit der „Energiewende“, mit „Bildungsgerechtigkeit“, „guter Arbeit“ und einer „vorsorgenden Finanzpolitik“. 57 Er schwächt den Sparkurs der Vorgänger-Koalition ab, indem er für die kommenden fünf Jahre nur Streichungen von 700 statt 1.400 Lehrerstellen vorsieht. Die Einsparungen bei den dänischen Schulen, den Frauenhäusern und beim Landesblindengeld wurden rückgängig gemacht. Auch die Kürzungen bei den kommunalen Zuschüssen sollen ab 2013 wieder zurückgeführt werden - zweckgebunden für den Ausbau der Krippenplätze. Dafür wurde der SPD-Wunsch nach einem beitragsfreien Kita-Jahr zu-

53 Zeitplan und Organisation der Koalitionsverhandlungen wurden anhand der Berichterstattung der Kieler Nachrichten, des Hamburger Abendblatts und des NDR ermittelt; auf Einzelbelege wird hier verzichtet.

54 Vgl. zur Einordnung dieser Begriffe Patrick Horst, Koalitionsbildungen und Koalitionsstrategien im neuen Fünfparteiensystem der Bundesrepublik Deutschland, in: ZPol, 20. Jg. (2010), H. 3 - 4, S. $327-408$, S. $332 \mathrm{f}$.

55 Siehe „Knackpunkte bei den Koalitionsverhandlungen“, in: kn-online.de vom 16. Mai 2012; „Erste Koalitionsrunde zur „Dänen-Ampel“ - Optimismus“, in: ebenda vom 16. Mai 2012; „Zwischen Wünschen und schroffer Realität", in: ebenda vom 17. Mai 2012 (Abruf am 17. Juni 2012).

56 Vgl. „Umfrage: Unternehmen halten „Dänen-Ampel“ für instabil“, in: kn-online.de, vom 10. Mai 2012; „Wirtschaft mahnt die Dänen-Ampel“, in: ebenda vom 16. Mai 2012; „Wirtschaft fordert von Kieler Regierung Tempo bei A20“, in: ebenda vom 16. Mai 2012 (Abruf am 17. Juni 2012).

57 Zitiert in: Bündnis für den Norden. Neue Horizonte für Schleswig-Holstein. Koalitionsvertrag 2012-2017 von SPD, Grünen und SSW, Kiel, Juni 2012, passim. 
rückgestellt. Die Subventionen für den Ökolandbau führte die Dänen-Ampel wieder ein. Zusätzliche Kürzungen oder Einnahmesteigerungen gegenüber den von der Vorgängerregierung bereits getroffenen Sparbeschlüssen enthält der Koalitionsvertrag nur wenige: Straßenbaumittel sollen gekürzt und der Erdölförderzins erhöht werden. Mehr auf Popularitätsdenn auf Einspareffekte zielte der Beschluss, die Bezüge der Kabinettsmitglieder zu kürzen. Dennoch will die Koalition die Vorgaben der Schuldenbremse einhalten und bis 2020 einen ausgeglichenen Haushalt vorlegen. ${ }^{58}$

In der Energiepolitik setzt die Koalition dem Land das Ziel, bis 2020 dreimal so viel Strom aus regenerativen Energien zu produzieren, wie es verbraucht. Neubauten von Kohlekraftwerken sollen verhindert werden. Auf dem Feld der Bildungspolitik einigten sich die Parteien im Grundsatz auf das achtjährige Abitur an Gymnasien und das neunjährige Abitur an den Gemeinschaftsschulen, sahen aber einen Bestandsschutz für die bestehenden G9Schulen vor. Die Lehrerausbildung soll reformiert und von den Schulformen abgelöst werden. In der Verkehrspolitik verständigten sich die Koalitionspartner darauf, die A 20 vorerst nur bis zum Anschluss an die A 7 bei Bad Bramstedt weiterzubauen. Den Landeszuschuss von 60 Millionen Euro für die Untertunnelung des Fehmarnbelts strich die Koalition, wohl wissend, dass das gesamte Projekt nur vom Bund und Dänemark gestoppt werden könnte. Die wichtigsten arbeitsmarktpolitischen Projekte umfassen eine Bundesratsinitiative zum Mindestlohn und ein Tariftreuegesetz zur Gleichstellung der Leiharbeiter mit der Stammbelegschaft. Im innenpolitischen Bereich will die neue Koalition das Wahlalter bei Landtagswahlen auf 16 Jahre senken, das Abschiebegefängnis in Rendsburg schließen und im Bundesrat gegen die Vorratsdatenspeicherung stimmen.

Schwieriger als die inhaltlichen Kompromisse, in denen sich alle drei Koalitionspartner gut wiederfinden konnten, war die Lösung der Konflikte hinsichtlich der Personalfragen und des Ressortzuschnitts. Die Eckpunkte, die der designierte Ministerpräsident Albig im Wahlkampf gesetzt hatte - mindestens vier Frauen im Kabinett, eine davon als Ministerin für Bildung und Wissenschaft die Flensburger Universitätspräsidentin Waltraud Wende, keine Vergrößerung des Kabinetts - waren nicht einfach mit den Interessen von gleich drei Partnern und den Präferenzen ihres Spitzenpersonals unter einen Hut zu bringen. Weil zusätzlich zum Ministerpräsidentenamt nur sieben Ministerposten zu vergeben waren, mussten erhebliche Neuverteilungen der Geschäftsbereiche vorgenommen werden (vgl. Tabelle 4): Die SPD erhielt neben dem Regierungschef vier Ministerien (Innen, Bildung, Wirtschaft, Soziales) und sieben Staatssekretäre. Die Grünen stellten mit Monika Heinold die Finanzministerin und ließen sich eigens für Robert Habeck ein Energiewendeministerium zuschneiden. Sie durften insgesamt drei Staatssekretäre nominieren. Beim SSW entschloss sich Fraktionschefin Anke Spoorendonk nach anfänglichem Zögern, als Ministerin für Justiz, Europa und Kultur in das Kabinett einzutreten. Ihr wurde ein Staatssekretär zugeordnet. Der Entscheidung nachgeholfen hatte der Umstand, dass der zunächst als Wirtschaftsminister gehandelte Lars Harms den Interessenvertretern der schleswig-holsteinischen Wirtschaft nicht vermittelbar war. ${ }^{59}$

58 Vgl. hierzu und zum folgenden Absatz „Dänen-Ampel nimmt Streichungen zurück“, in: Hamburger Abendblatt vom 4. Juni 2012, S. 12; „Der Koalitionsvertrag: Die wichtigsten Punkte“, in: ndr.de vom 4. Juni 2012 (Abruf am 27. Juli 2012); „Kernpunkte des Kieler Koalitionsvertrags“, in: kn-online.de vom 11. Juni 2012 (Abruf am 15. Juni 2012).

59 Siehe „Albig will Flensburger Universitätspräsidentin als Bildungsministerin“, in: kn-online.de vom 25. April 2012 (Abruf am 3. Mai 2012); „Spoorendonk will nun doch einen Ministerposten 


\begin{tabular}{|l|l|}
\hline Tabelle 4: Das am 13. Juni 2012 vereidigte schleswig-holsteinische Kabinett \\
\hline Ministerpräsident & Torsten Albig (49, SPD) \\
\hline $\begin{array}{l}\text { Minister für Energiewende, Landwirtschaft, Umwelt und ländliche } \\
\text { Räume (Stellvertretender Ministerpräsident) }\end{array}$ & Dr. Robert Habeck (42, Grüne) \\
\hline Ministerin für Justiz, Kultur und Europa & Anke Spoorendonk (64, SSW) \\
\hline Ministerin für Bildung- und Wissenschaft & Dr. Waltraud Wende (54, parteilos) \\
\hline Finanzministerin & Monika Heinold (53, Grüne) \\
\hline Innenminister & Andreas Breitner (45, SPD) \\
\hline Minister für Wirtschaft, Arbeit, Verkehr und Technologie & Reinhard Meyer (52, SPD) \\
\hline Ministerin für Soziales, Gesundheit, Familie und Gleichstellung & Kristin Alheit (44, SPD) \\
\hline Quelle: „Die Minister - Das Kabinett“, in: schleswig-holstein.de (Abruf am 28. Juli 2012). \\
\hline
\end{tabular}

Auf Seiten der Opposition musste die CDU zunächst mit den Folgen des Neuzuschnitts der Wahlkreise, die sie nicht zu ihren Ungunsten durchgeführt hatte, fertig werden: Weil sie 22 Wahlkreise gewonnen hatte, schafften es weder ihr Spitzenkandidat de Jager noch der bisherige Landtagspräsident Torsten Geerdts über die Landesliste in den Landtag. Die Fraktion bestätigte deshalb den bisherigen Fraktionschef Johannes Callsen im Amt. Nachdem Spekulationen ins Kraut schossen, wer für den Landesvorsitzenden sein Landtagsmandat zur Verfügung stellen müsste, beeilte sich de Jager zu versichern, dass für ihn niemand zurücktreten müsse und er die Partei auch aus der Parteigeschäftsstelle heraus führen könne. Fünf Tage nach der Wahl wurde eine mögliche Lösung in der Presse lanciert, wonach de Jager im Herbst 2013 für Callsen in den Landtag einziehen könne, falls dieser sich zu einer Kandidatur für den Bundestag als Nachfolger von Wolfgang Börnsen entschließe. Callsen selbst äußerte sich jedoch nicht zu dieser Spekulation. Die FDP wählte erwartungsgemäß Wolfgang Kubicki erneut zum Fraktionsvorsitzenden, der das Amt nun seit 1996 innehat. Zum stellvertretenden Vorsitzenden wurde Christopher Vogt gewählt, Parlamentarischer Geschäftsführer wurde der bisherige Sozialminister Heiner Garg. ${ }^{60}$

Besondere Aufmerksamkeit zog die neue Oppositionsfraktion der Piraten auf sich: Spitzenkandidat Torge Schmidt unterlag in der Wahl zum Fraktionsvorsitzenden Patrick Breyer; Schmidt wurde daraufhin Parlamentarischer Geschäftsführer. Breyer kündigte an, dass beide auf ihre Funktionszulagen verzichten und sich mit der Grunddiät eines Abgeordneten begnügen wollten. Mit zwei weiteren Initiativen meinten die Piraten, frischen Wind in den Kieler Landtag zu bringen: Erstens lehnten sie eine Pairing-Vereinbarung mit der Begründung ab, dass sich „Mehrheiten ohne Fraktionszwang aus der freien Überzeugung aller Volksvertreter bilden“ (Breyer) sollten. Zweitens traten sie dafür ein, die Sitzungen des Ältestenrats in Zukunft öffentlich abzuhalten. Dass ihre Demokratievorstellungen eher einem „klassisch-liberalen“ als einem modernen Parlamentarismusverständnis huldigten ${ }^{61}$, zeigte sich auch in der Nichtoffenlegung des Abstimmungsverhaltens bei der Wahl des Minister-

in Kiel“, in: Hamburger Abendblatt vom 24. Mai 2012, S. 16; Koalitionsvertrag, a.a.O. (Fn. 57), S. 58 - 60; dort auch die genaue Neuverteilung der Geschäftsbereiche.

60 Vgl. „Dänen-Ampel - wer wird was?“, in: Hamburger Abendblatt vom 9. Mai 2012, S. 14; „Neue Chance in einem Jahr“, in: ebenda vom 11. Mai 2012, S. 16; „Kubicki bleibt am [sic!] Amt“, in: kn-online.de vom 15. Mai 2012 (Abruf am 17. Juni 2012).

61 Dazu Eberhard Schuett-Wetschky, Zwischen traditionellem Parlamentsverständnis und moderner Parteiendemokratie: Gründe des latenten Verfassungskonflikte, in: ZParl, 34. Jg. (2003), H. 3, S. $531-549$. 
präsidenten. Die Piraten wollten nicht, so begründete Breyer die Entscheidung, „dass sich jemand unter Druck gesetzt fühlt oder sich rechtfertigen muss"62.

\section{Zusammenfassung}

(1) Bereits nach zweieinhalb Jahren wurde die schwarz-gelbe Koalition wieder abgewählt. Sechs von zehn Wählern waren mit ihrer Leistungsbilanz unzufrieden. Insbesondere die FDP wurde im Sog ihres miserablen Erscheinungsbildes auf Bundesebene katastrophal bewertet, was auch ein formidabler Wahlkampf ihres Spitzenkandidaten Kubicki nicht mehr wettmachen konnte. Die CDU musste im Sommer 2011 überraschend Jost de Jager als neuen Spitzenkandidaten nominieren, der es hinsichtlich seiner Sympathiewerte nicht mit dem Kontrahenten von der SPD aufnehmen konnte. Die CDU fuhr mit 30,8 Prozent ihr schlechtestes Wahlergebnis der Nachkriegsgeschichte ein, die FDP brachte es immerhin auf beachtliche 8,2 Prozent. Zusammen blieben sie mit nicht einmal 40 Prozent an Wählerstimmen weit entfernt von einer Regierungsmehrheit.

(2) Die Schwäche der Regierung konnte die größte Oppositionspartei SPD nur teilweise für sich nutzen. Für die angestrebte rot-grüne Koalition reichte der Stimmenzuwachs nicht aus. Die Sozialdemokraten verbesserten sich zwar um fünf Punkte, erreichten mit 30,4 Prozent aber nur das drittschlechteste Ergebnis seit 1947. Manche, vor allem die Anhänger des SPD-Landesvorsitzenden Stegner, machten dafür den wenig konfrontativen Wahlkampfstil Albigs verantwortlich. Die Umfragedaten zeigten jedoch, dass Albig nicht nur beliebter war als de Jager, sondern auch deutlich beliebter als Peter Harry Carstensen und Stegner im Wahlkampf 2009. Das schwache SPD-Ergebnis lag nicht an Albig, sondern am schlechten Image des Landesverbands, für das vor allem Stegner verantwortlich ist.

(3) Die Grünen erzielten bei einer Steigerung um 0,8 Punkte auf 13,2 Prozent ihr bisher bestes Ergebnis. Zwar blieben sie nach ihrem Höhenflug in den Wahlumfragen leicht hinter den Erwartungen zurück, angesichts der neuen Konkurrenz der Piraten war dies aber ein sehr achtbares Resultat, das sie zu einem Gutteil ihrem Spitzenkandidaten Habeck zu verdanken hatten, der ein ausgesprochener Sympathieträger im Lande ist.

(4) Die Piraten erreichten im Windschatten ihres bundespolitischen Höhenflugs stolze 8,2 Prozent und zogen mit sechs Abgeordneten nach Berlin und dem Saarland bereits in das dritte Parlament eines deutschen Bundeslandes ein. Sie waren die einzige Partei, die überwiegend aus bundespolitischen Motiven heraus gewählt wurde. Alle negativen Schlagzeilen im Wahlkampf konnten ihr nichts anhaben.

(5) Die Wahlbeteiligung sank gegenüber 2009 um 13,5 Punkte auf einen Tiefststand von 60,1 Prozent. Da die Landtagswahl 2009 zeitgleich mit der Bundestagswahl stattgefunden hatte, wäre 2005 ein besserer Vergleichspunkt. Der Rückgang von 6,4 Punkten gegenüber 2005 war immer noch stattlich, zumal der Ausgang der Wahl bis zuletzt offen war.

62 Vgl. zum Ganzen „Piraten schassen ihren Kapitän“, in: Hamburger Abendblatt vom 22. Juni 2012, S. 14; „Piraten lehnen parlamentarischen Brauch ab - Kein Pairing mit dem SSW“, in: kn-online. de vom 24. Mai 2012; „Piraten wollen Vertraulichkeit des Ältestenrats ignorieren“, in: ebenda vom 31. Mai 2012; „Die Neuen erobern den Landtag“, in: ebenda vom 5. Juni 2012 (Abruf am 17. Juni 2012); „Albig rührt Carstensen zu Tränen“, in: Hamburger Abendblatt vom 13. Juni 2012, S. 14. 
(6) Die Regierungsbildung lief im Grunde schon am Wahlabend auf die Dänen-Ampel zu; nur CDU-Spitzenkandidat de Jager verwehrte sich dieser Einsicht noch einige Tage. Die Bildung dieser in Deutschland neuartigen Koalition ging mit 38 Tagen zügig vonstatten. Im Koalitionsvertrag können sich alle Parteien mit ihren inhaltlichen Positionen wiederfinden; die Ressortverteilung ist gemessen an der Stärkerelation der Koalitionspartner sehr gerecht. Die Grünen, die mathematisch ein klein wenig zu kurz kamen, wurden mit zwei Schlüsselministerien (Energiewende, Finanzen) entschädigt.

(7) Den drei Regierungsparteien stehen mit CDU, FDP und den Piraten auch drei Oppositionsparteien gegenüber. Die Piraten wollen allerdings sachorientiert entscheiden und stehen deshalb einer Zusammenarbeit mit der Regierung offen gegenüber. Die innenpolitischen Beschlüsse der Koalition (Senkung des Wahlalters auf 16 Jahre, Ablehnung der Vorratsdatenspeicherung) ermöglichten es wahrscheinlich zwei Piraten, bei der Wahl des Ministerpräsidenten für Albig zu stimmen.

\title{
Die nordrhein-westfälische Landtagswahl vom 13. Mai 2012: Von der Minderheit zur Mehrheit
}

\author{
Stefan Bajohr
}

Die Wahl zum Landtag am 13. Mai 2012 kam nicht unerwartet, aber überraschend. Seit der Bildung einer rot-grünen Minderheitsregierung im November 2010 hatten Medien und Politik spekuliert, wie lange sich diese Koalition halten könne. Manche meinten, dass sie bereits daran scheitern würde, einen Haushalt durch das Parlament zu bringen. Sie sahen sich getäuscht. Die relative Stabilität der Landesregierung beruhte darauf, dass die Opposition ihre Mehrheit nicht in einem konstruktiven Misstrauensvotum gegen die Ministerpräsidentin bündeln konnte und mit Blick auf ihre Umfragewerte kein Interesse hatte, Neuwahlen herbeizuführen.

Als es schließlich doch zur Landtagsauflösung kam, waren ausgerechnet die Parteipolitiker* am meisten verblüfft. Einige von ihnen hatten gezündelt, aber den Brand nicht vorausgesehen.

\section{Die Ausgangslage}

Nach der für Schwarz-Gelb verloren gegangenen Landtagswahl vom 9. Mai 2010 wagte die Sozialdemokratin Hannelore Kraft nach anfänglichem Zögern das Experiment einer rotgrünen Minderheitsregierung. Mit 90 Stimmen von SPD und Grünen bei Enthaltung der Linken (elf Stimmen) und 80 Gegenstimmen von CDU und FDP wurde sie zur Minister-

* Dieser Aufsatz wurde ursprünglich in geschlechtsneutraler Sprache verfasst. Die ZParl-Redaktion samt ihrer Chefredakteurin teilt die Auffassung des Autors, dass Sprache Wirklichkeit gestaltet. Sie meint aber, dass die geschlechtsneutrale Fassung schwerer lesbar sei und bevorzugt deshalb die traditionelle Schreibweise. 